\title{
A Study on Anti-Clone and Authenticity Verification Technique for Ceramics: Pilot testing of New Glass Phosphor with Two Optical Features
}

\author{
Masaki FUJIKAWA ${ }^{\mathrm{a},{ }^{*}}$, Mariko HARA ${ }^{\mathrm{a}}$, and Shingo FUCHI ${ }^{\mathrm{b}}$ \\ ${ }^{a}$ Kogakuin University, Nishi-Shin-jyuku 1-24-2, 163-8677, JAPAN \\ ${ }^{\mathrm{b}}$ Aoyama Gakuin University, Fuchinobe 5-10-1, 252-5258, JAPAN
}

\begin{abstract}
Multi-modal artifact metrics as an anti-counterfeiting technique is created based on the concept of multi-modal biometrics. Giving two or more characteristic information to the artifact can improve the certainty of authenticity and increase counterfeiting difficulty. To give two-optical feature information (color hue and emission intensity) into ceramic products, we developed a new type of glass phosphor. It is a novel approach, because there were few reports on up-conversion phosphors having different color hue and emission intensity at each observation point on the material by infrared light excitation. By welding a small amount of phosphor powder onto the surface of ceramics, the certainty of authenticity and difficulty of counterfeiting could be enhanced than an existing method we proposed. Our study established an appropriate blending ratio of two rare earth oxides for making glass phosphor having two optical features. Welded glass phosphor powder could withstand high temperature (with 800 degrees Celsius for decorating firing and 1230 degrees Celsius for glaze firing) and emit different color hue and intensity after the firing. This shows our method could have a potential to be used for the purpose of verifying the product's authenticity.
\end{abstract}

Keywords: anti-clone, authenticity, ceramics, glass phosphor, multi-modal artifact metrics, transfer paper.

\section{Introduction}

\subsection{Background and Target}

Ceramic products made by prestigious as well as well-known potteries or brand holders are highly popular and can be sold at a high price as high-quality accessories. Counterfeiters manufacture imitations of these products and sell them to people with low appraisal skills ${ }^{(1)}$. The existence of copied ceramic products cannot be overlooked, because the distribution of counterfeit products not only violates the intellectual property rights of potteries and brand holders but cost them a share of income that they could have otherwise obtained. Besides, financial and psychological damages can also be made to consumers who purchase them unknowingly.

We have developed a technique that can verify the product's authenticity mechanically with high accuracy to limit people with low appraisal skills to purchase imitations unknowingly. This technique is defined as the artifact metrics ${ }^{(2)}$ because it makes it difficult for forgers to manufacture the genuine product's clone. In our previous research ${ }^{(3)}$, we focused on a transparent glass phosphor that emits one peak wavelength in response to infrared (IR) light excitement. We also proposed a technique to weld small amount of phosphor powder onto the surface of products. It has three features as shown as follows: (1) The powder has low toxicity and does not affect the product's colors/patterns. (2) The characteristic information (emission intensity) used for verification of authenticity can be extracted from each product in a non-contact manner. (3) It is difficult to manufacture clones using the extracted characteristic information from genuine products.

We also have proposed a concept called the multi-modal artifact metrics hinted by multi-modal biometrics ${ }^{(4)}$ to enhance the accuracy of authenticity and difficulty of counterfeiting by adding over another characteristic information to the artifact. In addition, as a countermeasure of counterfeiting valuable cards (such as credit cards), we proposed a method of adding both sheet resistance (as characteristic electrical information) and phosphor emission (as optical feature information) to synthetic resin cards. ${ }^{(5)}$ 
To date, we have been trying an approach of applying the multimodal artifact metrics to ceramics and extracting several characteristic optical information from them. In this paper, we describe the development of new glass phosphor for this optical approach. By welding small amount of phosphor on the surface of such ceramic products, the resultant film is expected to give two optical feature information (hue and emission intensity). Therefore, the accuracy of authenticity and difficulty of counterfeiting could be enhanced compared to our previous study findings (3). Details will be described in Section 2.

This paper is organized as follows: In the remaining part of Section 1, the artifact metrics and multi-modal artifact metrics are outlined. Section 2 provides an outline of our idea while the fabrication process of glass phosphor is described in Section 3. In Section 4, we show another procedure of welding glass phosphor powder onto the surface of ceramics. Considerations based on the experimental results are then given in Section 5, while Section 6 finally draws the conclusions.

\subsection{Overview of the Artifact Metrics}

Basically, the concept of artifact metrics is similar to biometrics. Each artifact has unique characteristic information. Artifact's authenticity can be verified by extracting characteristic information from target artifact and comparing it with the registered feature information. The difficulty of counterfeiting is established based on technological evidence, by which the characteristic information held by the genuine product is difficult to copy. This characteristic information is formed spontaneously and randomly during the manufacturing process, and cannot be formed intentionally even by manufacturers of genuine products. Hence, the difficulty of counterfeiting cannot be decreased if the mechanism of forming characteristic information is accessible to the public.

Microscopically, each artifact has different characteristic information, such as tiny roughness and color shade. However, specifying the area photographed by registration phase and the area shot by verification phase is time consuming, because the area observed using a microscope is quite small ${ }^{(3)}$. Hence, in artifact metrics, a method is utilized to form unique and easy-to-extract characteristic information in the artifact. Specifically, additives having physical features are added during the manufacturing process. The particles are distributed randomly and fixed in the artifact. The distribution degree reflects the characteristic information. Sensing devices
Table 1. Physical characteristics \& extracted information.

\begin{tabular}{c|l}
\hline $\begin{array}{c}\text { Physical } \\
\text { characteristics }\end{array}$ & \multicolumn{1}{|c}{ Extracted feature information } \\
\hline $\begin{array}{c}\text { Optical } \\
\text { characteristics }\end{array}$ & $\begin{array}{l}\text { Particles' optical characteristics (reflection, } \\
\text { transmission, infraction, and fluorescence) and their } \\
\text { degree of distribution reflect the characteristic } \\
\text { information, which is extracted by sensors that can } \\
\text { detect light intensity. }\end{array}$ \\
\hline $\begin{array}{c}\text { Magnetic } \\
\text { characteristics }\end{array}$ & $\begin{array}{l}\text { Particles' magnetic characteristics (attraction and } \\
\text { repulsive force) and their degree of distribution } \\
\text { reflect the characteristic information, which is } \\
\text { extracted by sensors that can detect a change in } \\
\text { magnetism. }\end{array}$ \\
\hline $\begin{array}{c}\text { Electrical } \\
\text { characteristics }\end{array}$ & $\begin{array}{l}\text { Particles' electric characteristics (electrical charge) } \\
\text { and the degree of distribution reflect the } \\
\text { characteristic information, which is extracted by } \\
\text { sensors that can detect the quantity of electric } \\
\text { charge. }\end{array}$ \\
\hline $\begin{array}{c}\text { Vibration } \\
\text { characteristics }\end{array}$ & $\begin{array}{l}\text { Particles' vibration characteristics (sonic waves) and } \\
\text { the degree of distribution reflect the characteristic } \\
\text { information, which is extracted by sensors that can } \\
\text { detect sonic waves. }\end{array}$ \\
\hline \hline
\end{tabular}

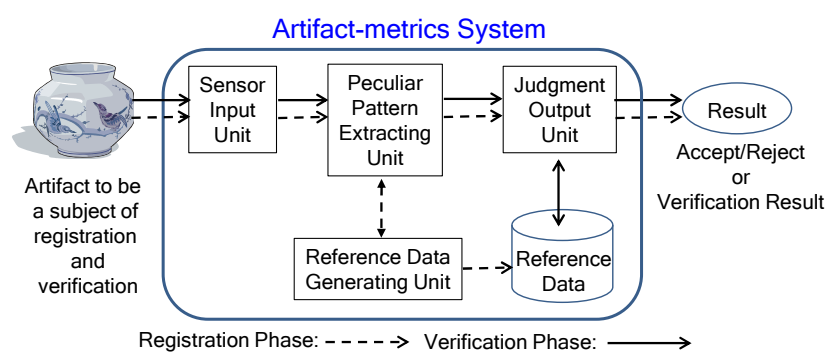

Fig. 1. Overview of the artifact metric system.

which can extract the physical features of additives are used when extracting characteristic information.

Table 1 shows the physical characteristics of materials added in the manufacturing process and the characteristic information extracted from them. Fig. 1 shows an overview of the system (artifact metric system) that uses artifact metrics. Two phases are almost the same as those of biometrics. In this system, characteristic information that is extracted from each artifact before it is shipped is stored in a secure database. To verify the authenticity of an artifact, the system extracts the characteristic information from it and compares the information with that of the registered feature in the secure database.

\subsection{Overview of Multi-modal Artifact Metrics}

Characteristic information extracted from artifacts can be changed, depending on environmental circumstances during extraction (such as temperature, humidity, and position of artifacts relative to sensing devices). However, even in such situations, the artifact metric system should be able to verify authenticity stably and correctly, using the 
Table 2. Comparison between two approaches.

\begin{tabular}{c|l|l}
\hline \hline & \multicolumn{1}{|c|}{ Approach 1 } & \multicolumn{1}{c}{ Approach 2 } \\
\hline Advantages & $\begin{array}{l}\text { Low probability of } \\
\text { affecting the artifact's } \\
\text { moldability and physical } \\
\text { strength. }\end{array}$ & $\begin{array}{l}\text { Increases the number of } \\
\text { extractable feature } \\
\text { information. }\end{array}$ \\
\hline Drawbacks & $\begin{array}{l}\text { Limited with regard to } \\
\text { the number of extractable } \\
\text { feature information. }\end{array}$ & $\begin{array}{l}\text { High probability of } \\
\text { affecting the artifact's } \\
\text { moldability and physical } \\
\text { strength. }\end{array}$ \\
\hline \hline
\end{tabular}

strong correlation between the characteristic information registered in the database and the information features extracted during verification.

There is an approach to increase the number of characteristic information to find strong correlations between both registered and extracted information ${ }^{(3)}$. This approach is named "multi-modal artifact metrics (5)" because various characteristic information is used. This approach can be further categorized into two methods. The first one (approach 1) focuses on additives having one physical feature and extracts two or more types of characteristic information from the artifact. For example, in our previous paper ${ }^{(3)}$, especially in the "consideration" section, we focused on the optical feature of glass phosphor and proposed another idea to utilize two distributions (emission spectrum and emission intensity distribution) as the characteristic information. These distributions can reflect the particle size and degree of dispersion of glass phosphor welded on the surface of ceramic products.

The second method (approach 2) adds two or more additives having different physical features to the artifact, from which two or more types of characteristic information can be extracted. For example, in our previous study ${ }^{(5)}$, we proposed an idea of creating thin films inside synthetic resin cards (used for valuable cards) using a conductive polymer paint having electrical features, and infrared up-conversion phosphor powder having optical features. This method utilizes emission intensity and sheet resistance which reflect the size and dispersion of phosphor particles in thin films. This information could be used as the characteristic information.

The differences of both methods are listed in Table. 2 . Although approach 1 is limited by the amount of characteristic information extracted from the artifact, it could reduce the quantity of materials for the total amount of artifacts. Approach 2 could increase more characteristic information than the former one. However, it could also affect the artifact's moldability and physical strength, because the material quantity for the total amount of artifacts increases.

\section{New Approach}

\subsection{Outline of Another Method and Challenges}

In this section, we outline another multi-modal artifact metrics method for ceramics as proposed in the "considerations" section in our previous paper ${ }^{(3)}$, and its challenges for practical use. The purpose of this paper is to propose the artifact metrics (single modal) using emission intensity distribution (See Fig. 2) as the characteristic information resulting from glass phosphor particles welded on the surface of products. This study also proposes another method to use both the emission intensity distribution and emission spectrum distribution as feature information to verify the stability of product's authenticity. This method could be identified as the multi-modal artifact metrics because it utilizes multiple characteristic information. It, thus, can enhance the accuracy of authenticity and difficulty of counterfeiting.

In the above study, a glass phosphor emits a peak wavelength at $1000 \mathrm{~nm}$ by optical excitation (808 $\mathrm{nm}$ of IR light). This is called a down-conversion phosphor, because the emission wavelength shifts to the opposite direction (or long wavelength side) of the optical excitation wavelength. The challenge of this method is the requirement of complex optical system having high price, such as near-infrared hyper spectrum cameras ${ }^{(6)}$. Although this system can extract two distributions in the infrared wavelength band, it provides huge implications on the device configuration of the artifact metric system and manufacturing cost. It would be a barrier for practical use.
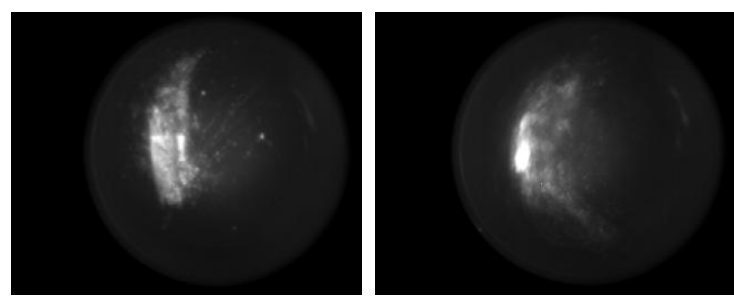

Fig. 2. Emission intensity distribution images.

\subsection{Solutions}

To eliminate the barrier for practical use and realize the method (the multi-modal artifact metrics) for ceramic products, we propose an approach of putting the light emission of phosphor in the visible light band instead of the IR band. In concrete, a phosphor with visible light emission by optical excitation was developed and a light emission was observed using visible camera. 

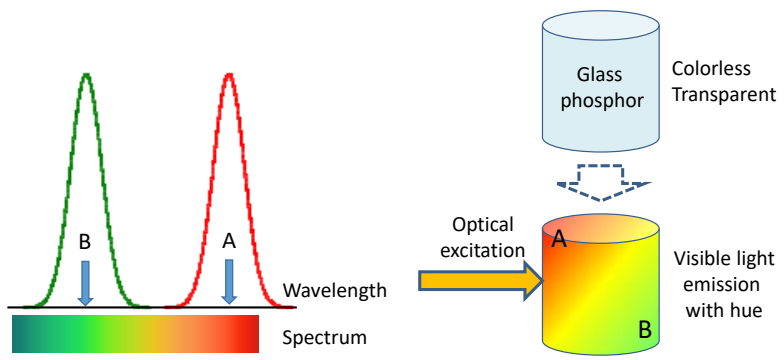

Fig. 3. Two emission peaks of wavelength (left) and visible light emission with optical excitation (right).

As shown in Fig. 3, the former idea could be realized by developing transparent glass phosphor that provides two peak wavelengths in visible light band. The difference of emission colors could be observed by mixing light colors (See Fig. 3: right) if the heights of peak wavelengths are different at each observation point. According to our previous study ${ }^{(3)}$, glass phosphor as an additive derives without toxicity and has a high affinity for glaze as well as paint due to the same glassy component. The ceramic's patterns/colors could not be affected if the amount of phosphor is small. Visible cameras have simple system composition and are cheaper than infrared hyper spectrum cameras; therefore, cost would not be a barrier for practical use. The emission spectrum distribution (the wavelength and corresponding color) and emission intensity distribution (the light's strength) could also be captured in visible light band.

\subsection{Features of the Novel Glass Phosphor}

Unlike the phosphor described in our previous study ${ }^{(3)}$, the novel glass phosphor shows different light emissions and intensity based on the locations where the excitation light is irradiated (See Fig. 3 and 4). Red color could be observed at observation point $\mathrm{A}$, while green color could be observed at observation point $\mathrm{B}$. The yellow color means that red and green light are emitted at that point. The emission difference based on the observation point occurs because of the distance between ions and the ratio difference of the number of ions from multiple rare earth oxides contained in glass phosphor.

Similar to our previous study ${ }^{(3)}$, we chose to weld the glass phosphor powder onto the surface of ceramic products. Two types of feature information, namely, emission spectrum distributions (hue) and emission intensity distributions, could be extracted by photograph, when the unevenness of distance between ions and the number of ions were retained in the powder.
Generally, the up-conversion phosphor (emission wavelength shifts to the short wavelength side rather than the optical excitation wavelength) was developed with the aim of having one sharp peak wavelength (or single color), which exhibits one-peak wavelength in the visible light band and requires infrared light as the light source. Because so far, developing an up-conversion phosphor with a two-peak wavelength by changing the ratio of rare earth oxides has been rarely reported, our approach seems to be novel. An approach exists for developing the down-conversion glass phosphor having the same features, which use an ultraviolet (UV) light source. However, UV light could affect the colors/pattern of the ceramic during the irradiation on the surface of ceramic products. Hence, in this study, we developed an up-conversion phosphor which utilizes an infrared light source.

\section{Experiments}

\subsection{Searching Method for finding Ratio of Rare Earth Oxide}

To have two emission peak wavelengths (red and green light emission) as shown in Fig.3, we used $\mathrm{Er}_{2} \mathrm{O}_{3}$ for red and green light emissions as well as $\mathrm{Yb}_{2} \mathrm{O}_{3}$ for absorbing IR light and transmitting the light energy to Erbium ions as excitation energy. The glass phosphor can be made by putting regents into a crucible, melting them in a furnace (about 1,230 degrees Celsius for several tens of minutes) and cooling it rapidly after extracting from the furnace.

These compositions could generate points in the glass phosphor, where the numbers of Ytterbium and Erbium ions and the distance between them would be spontaneously uneven during the synthesis process. This phenomenon could provide different emission spectra (hue) and emission intensity at varied observation points. The emission spectra (hue) can be changed by the distance between ions and the ratio of the numbers of Ytterbium and Erbium ions, while emission intensity was controlled by the number of Erbium ions.

In our experiment, we made candidates of glass phosphor by changing the weight of each $\mathrm{Er}_{2} \mathrm{O}_{3}$ and $\mathrm{Yb}_{2} \mathrm{O}_{3}$, while the mass of the glass phosphor was fixed as 10 grams. If the difference of the hue and emission intensity could be observed on the surface of the product by naked eyes, the ratio was gradually changed to be close to the appropriate value, based on the weight of the rare earth oxide contained 


\section{$\underline{\mathrm{XYb}_{2} \mathrm{O}_{3}}-\underline{\mathrm{YEr}_{2} \mathrm{O}_{3}}-\underline{\left(10 \mathrm{ZnO}-45 \mathrm{Sb}_{2} \mathrm{O}_{3}-45 \mathrm{GeO}_{2}\right)}$}

Fig. 4. Chemical formula of targeted glass phosphor.

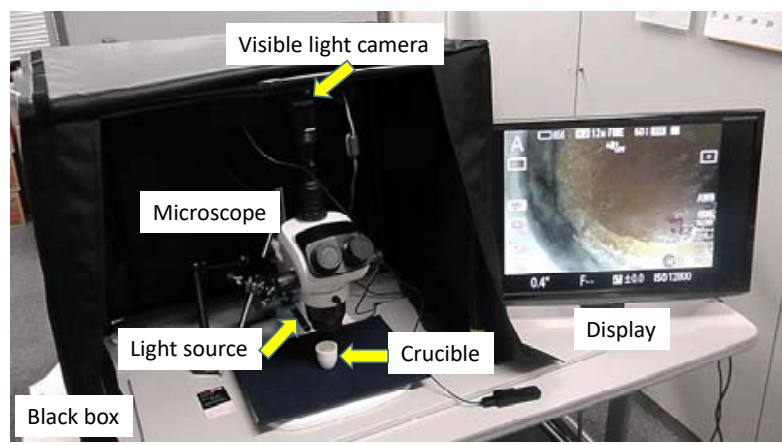

Fig. 5. Visible light emission observation system.

in the candidate. A concrete process is shown below. In this process, $10 \mathrm{ZnO}-45 \mathrm{Sb}_{2} \mathrm{O}_{3}-45 \mathrm{GeO}_{2}$ was used as the base material glass.

(1) Define the value of mol \% of each Ytterbium oxide and Erbium oxide as $\mathrm{X}$ and $\mathrm{Y}$, respectively, and calculate the corresponding weight. $\mathrm{X}$ and $\mathrm{Y}$ correspond to the red colored letter indicated in Fig. 4.

(2) Put the weighed reagents into the crucible and melt it in the furnace. Take the melted reagents from the furnace and cool it rapidly.

(3) Regarding a candidate A, if the difference of the hue and emission intensity could be seen at any observation point while irradiating by IR light, adjust $\mathrm{X}$ and $\mathrm{Y}$ to find the existence of a candidate $\mathrm{B}$ which provide more intense light emission than $\mathrm{A}$.

(4) Return to step (1).

In our experiment, candidates were set in a system (See Fig. 5) to photograph visible light emission. We used a commercially available single-lens reflex camera (SONY alpha 7s) with an IR cut filter.

\subsection{Experiment 1}

We used $10 \mathrm{ZnO}-45 \mathrm{Sb}_{2} \mathrm{O}_{3}-45 \mathrm{GeO}_{2}$ as the base material glass and conducted an experiment by following the above-mentioned process to determine the appropriate ratio of the two types of rare earth oxides. This material glass could enhance the emission intensity of glass phosphor based on our past experiments. Fig. 6 shows the glass phosphor which showed the most noticeable difference of the hue and emission intensity. This phosphor contains 5.0 mol $\%$ of Ytterbium oxide and $0.08 \mathrm{~mol} \%$ of Erbium oxide. Because the viscosity was higher than other melted

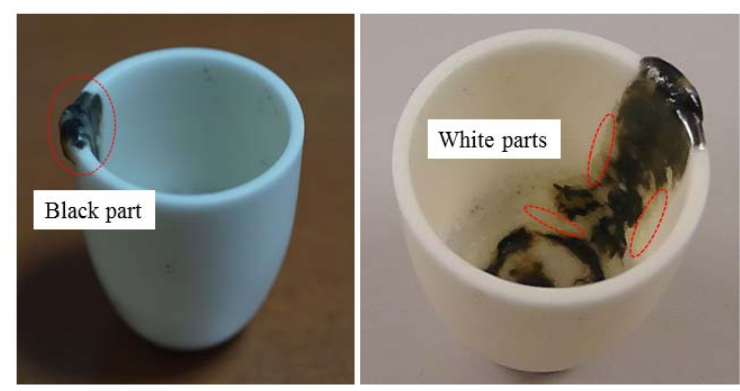

Fig. 6. A glass phosphor made in the experiment 1.

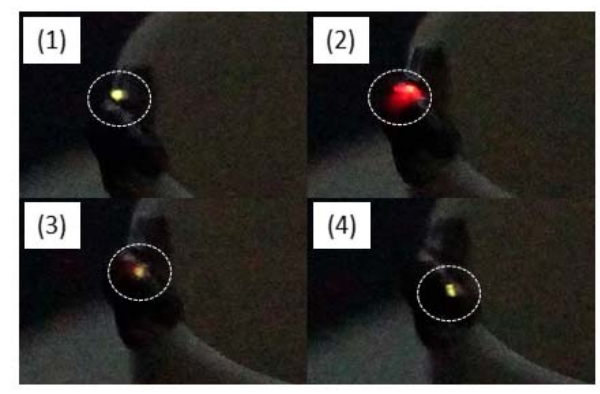

Fig. 7. Visible light emission (Black part).

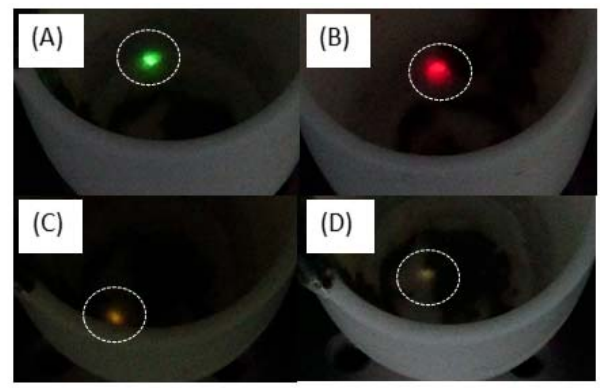

Fig. 8. Visible light emission (White part).

materials, it could not be taken from the crucible. The black-colored phosphor was welded on the side, while the white-colored phosphor was adhered on the bottom, respectively. The visible light emission in the black and white parts are shown in Fig. 7 and 8. The difference of the emission spectrum (hue) and emission intensity could be seen at each observation point. Hence, a glass phosphor having different hue and emission intensity at any observation point was successfully created.

\subsection{Experiment 2}

The aim of this experiment was to establish whether another glass phosphor having different hue and emission intensity at any observation point could be created utilizing the ratio of rare earth oxide determined in the previous section, even when the base material glass was replaced with another one. We had two intentions: One was to confirm the invariance of the ratio of rare earth oxides. The other one was whether the glass phosphor's color could be changed. The color and transparency of glass phosphor 


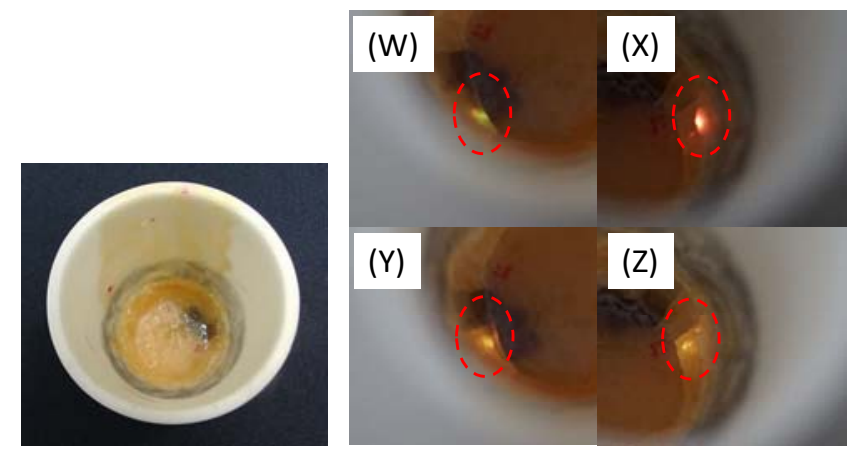

Fig. 9. A glass phosphor made in the experiment 2 (left) and difference of the light emission (right).

were determined by the composition of the base material glass. Therefore, the transparency could be increased if the color is changed.

In the experiment, we changed the composition of the base material glass from $10 \mathrm{ZnO}-45 \mathrm{Sb}_{2} \mathrm{O}_{3}-45 \mathrm{GeO}_{2}$ used in experiment 1 to $10 \mathrm{Bi}_{2} \mathrm{O}_{3}-45 \mathrm{Sb}_{2} \mathrm{O}_{3}-45 \mathrm{GeO}_{2}$. Because the color of the phosphor having $\mathrm{Bi}_{2} \mathrm{O}_{3}$ is likely yellow, the phosphor created in this experiment had a yellow color. The created glass phosphor is shown in Fig. 9 (left). Although it contained the same ratio of rare earth oxides as in experiment 1 , it showed the yellow color as we predicted. The light emission at each observation point is shown in Fig. 9 (right). The emission spectrum (hue) and emission intensity were different. Hence, the ratio of rare earth oxide could be applicable to another base material glass. There is a possibility of increasing the transparency of the glass phosphor.

\section{Applicability Assessment}

\subsection{Welding Powder onto the Pottery Plate (Type 1)}

In general, the biscuit of ceramic products is coated by liquid glaze. After the glaze dried, each product was baked in the furnace. The surface of the product became smooth because it was covered by the glaze with glassy substance. As the method of welding glass phosphor powder onto ceramic products, coating biscuit products by liquid glaze with phosphor powder seemed to be better. However, in this assessment, we welded the powder using the following method as a matter of convenience, because the amount of phosphor powder was not enough to do so.

We baked two plates with and without phosphor powder, respectively, in the furnace. The temperature in the furnace was increased up to 1,230 degrees Celsius in 8 hours. After keeping this degree for 10 minutes, the

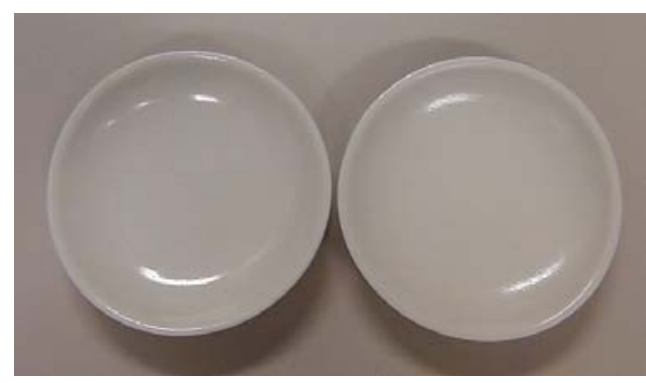

Fig. 10. A plate without powder (left) and with it (right).
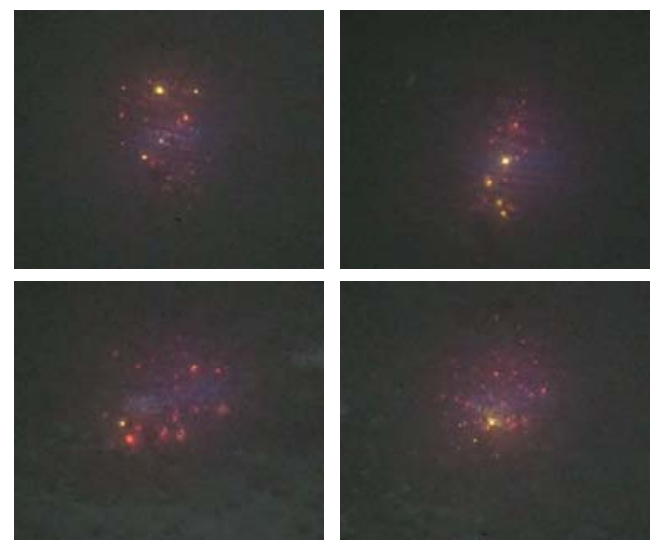

Fig. 11. Difference of the light emission at each observation point (2.5 magnification, ISO 40000).

(1) Make the dried glaze powder. This glaze turns into transparent glassy material after firing.

(2) Coat the biscuit plate by liquid glaze. After the glaze has dried, adhere the glass phosphor powder $(0.05$ grams) onto the surface of the plate by tip of a finger.

(3) Sprinkle the small amount of glaze powder over the area with phosphor powder and smooth the surface by tip of a finger.

temperature was decreased to the room temperature in 24 hours. The plates with and without glass phosphor powder are shown in Fig. 10. They had indistinguishable differences in appearance. We photographed the light emission at four observation points (See Fig. 11) using the system as shown in Fig. 5. The emission spectrum (hue) and emission intensity were different at each point. The glass phosphor can withstand the high temperature while keeping its optical feature.

\subsection{Welding Powder onto the Pottery Plate (Type 2)}

In the previous section, we mixed small amount of glass phosphor powder with liquid glaze, coated the biscuit of ceramic products by the glaze and baked them with high temperature in order to verify the approach's applicability of containing glass phosphor powder into the glassy 


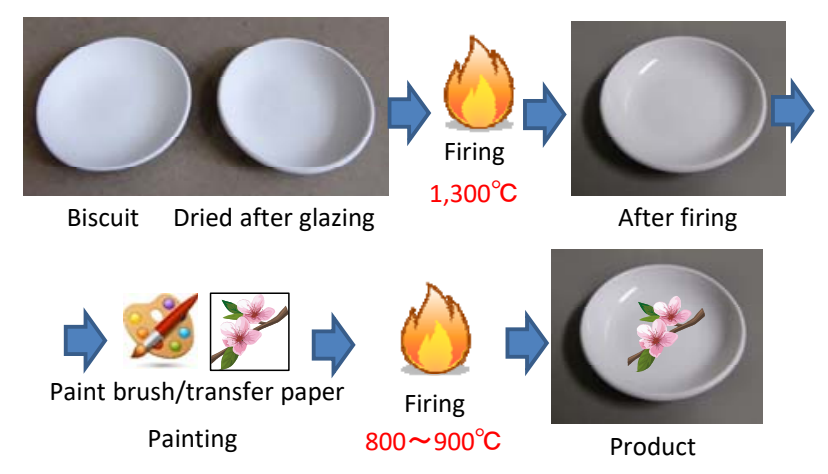

Fig. 12. Two firing processes through manufacturing.

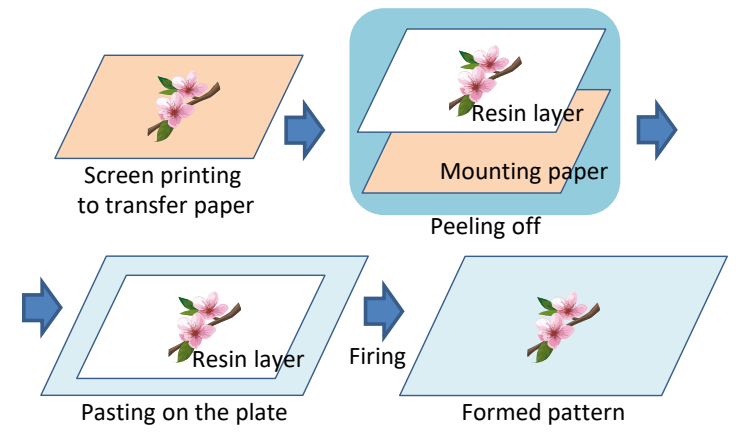

Fig. 13. Pattern baking method using transfer paper.

substance which covers ceramic product. In this section, we conduct an experiment of welding small amount of glass phosphor onto the surface of the glassy substance in order to verify another approach's applicability of containing glass phosphor powder into the patterns depicted on the surface of the ceramics (See Fig. 12). We use transfer paper without any printed patterns in this experiment. In general, as shown in Fig. 13, transfer paper is consisted of mounting paper and resin layer (with glass component which melts at about 800 degrees Celsius) and patterns are printed on the surface of the layer. These patterns are printed by the paint with same glass component. The resin layer peeled off from the mounting paper is pasted onto the surface of glassy substance. Although the layer burns out at 800 degrees Celsius, patterns are formed on the surface of glassy substance as glass component welds with the substance.

We conducted the experiment as follows: Adhere the small amount of glass phosphor onto the surface of glassy substance. Peel off the resin layer without printed patterns from mounting paper, paste it onto the glassy substance to cover glass phosphor powder and dry it well. Put the plate into the furnace, raise the temperature up to 800 degrees Celsius in 5 hours. Decrease the temperature down to room temperature in 24 hours.

The plates with and without glass phosphor powder are shown in Fig. 14. They had indistinguishable

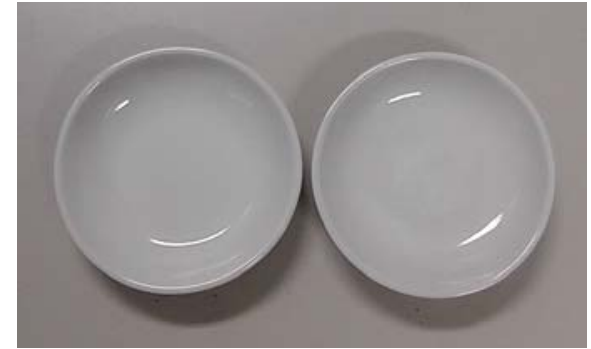

Fig. 14. A plate without powder (left) and with it (right).

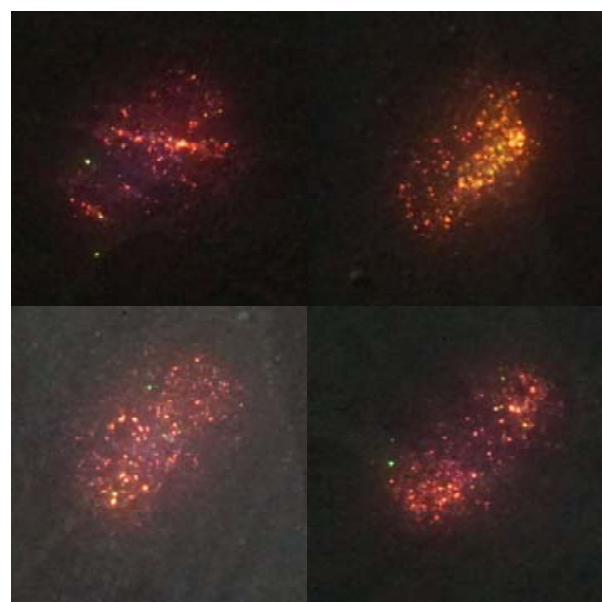

Fig. 15. Difference of the light emission at each observation point (2.5 magnification, ISO 40000).

differences in appearance. We photographed the light emission at four observation points (See Fig. 15) using the system as shown in Fig. 5. The emission spectrum (hue) and emission intensity were different at each point. The emission intensity is stronger than the experimental results described in the previous section. We assume the reasons of this phenomenon, such as changing the distance between ions could be seen at 1,230 degrees Celsius, receiving chemical effect from the component of glaze while firing.

\section{Considerations}

\subsection{Safety of the Phosphor}

While small amounts of rare earth elements are used to produce the glass phosphor, no apparent toxicity can be observed from the rare earth elements ${ }^{(7)}$. Materials of the supporting glass (oxide glasses of boron oxide system, phosphoric acid system, and boron anhydrate system) are stable oxides that are incombustible and insoluble. Their toxicities are very low. For example, lead oxide is contained in high quality transparent glass called crystal glass. Such glass is used as tableware and brings a serious safety concern. Furthermore, glass phosphor that is the compound between rare earth elements and the supporting glass is an 
Table 3. Comparison between glass phosphor and others.

\begin{tabular}{ll}
\hline \hline \multicolumn{1}{c}{ Material } & \multicolumn{1}{c}{ Consideration } \\
\hline $\begin{array}{l}\text { Transparent magnetic } \\
\text { substance }\end{array}$ & $\begin{array}{l}\text { The magnetizing process is required after } \\
\text { firing because the magnetic force wanes at } \\
\text { high heat during firing. }\end{array}$ \\
$\begin{array}{c}\text { Transparent conductive } \\
\text { material }\end{array}$ & $\begin{array}{l}\text { It loses transparency above } 600 \text { degrees C } \\
\text { during firing. }\end{array}$ \\
$\begin{array}{l}\text { Quartz with rare earth } \\
\text { It cannot melt 1200 to 1300 degrees C } \\
\text { oxide }\end{array}$ & $\begin{array}{l}\text { during firing. It melts above 1550 degrees } \\
\text { It melts 1200 to 1300 degrees C and } \\
\text { Glass phosphor }\end{array}$ \\
doesn't lose its function (transparency and \\
IR emission) after firing.
\end{tabular}

incombustible and insoluble oxide glass. As mentioned above, glass phosphor added to the artifact is less risky in affecting human health and environment. However, care should be taken to prevent inhalation and adhesion of the particles to naked eyes in handling the powders.

\subsection{Advantages of the Glass Phosphor}

As shown in Fig. 9 (left), the current glass phosphor was pale yellow. The colors/patterns of the ceramic products could be affected if the amount of phosphor powder welded on the surface of the product was increased. However, the amount of the powder was low. It seems to be difficult to distinguish the existence of the glass phosphor by naked eyes. Although some transparent materials exist, each candidate has application problems to for the porcelain products (See Table. 3). This shows the advantage of glass phosphor for practical use.

\section{Conclusions}

We developed the glass phosphor which shows two peak wavelengths in visible light band by optical excitation to apply the multi modal artifact metrics for ceramic products. Two optical feature information (hue and emission intensity) could be extracted from any observation point by welding glass phosphor powder onto the ceramic product during manufacturing process. The authenticity could be verified using registered images stored in the artifact metric system because two characteristic information was recorded as images in verification phase. In the experiment of creating glass phosphor, we made two types of glass phosphor by changing blending ratio of Ytterbium and Erbium oxides to estimate the appropriate component of phosphor. Therefore, we successfully made the glass phosphor having different hue and emission intensity at any observation point. This result could support the fact that the glass phosphor having the same optical feature could be made, even if the base glass material changed.
Two types of pottery plates were made (with and without glass phosphor) in our experiment. No big difference in appearance can be seen between them. However, the difference of the hue and emission intensity at each observation point can be shown by irradiating the IR light onto the plate with welded phosphor powder. The patterns/colors of the product were not affected by the small amount of phosphor powder. The phosphor's function can withstand high temperature and the component of glaze.

\section{Acknowledgment}

This work was supported by JSPS KAKENHI Grant Number 18K11302 and The Kazuchika Okura Memorial Foundation.

\section{References}

(1) MeissenPorcelain.com. (July 2013). Meissen Porcelain Reproductions and Fakes. [Online]. Available: http://meissenporcelain.com/meissen-porcelain-reprod uctions/

(2) T. Matsumoto, M. Hoga, Y. Ohyagi, M. Ishikawa, M. Naruse, K. Hanaki, R. Suzuki, D. Sekiguchi, N. Tate, and M. Ohtsu, "Nano-artifact metrics based on random collapse of resist," Nature Science Reports 4, Article number 6142, pp. 1-5, 2012.

(3) M. Fujikawa, F. Oda, K. Moriyasu, S. Fuchi, and Y. Takeda, "Development of the New Artifact-metircs Technology for Valuable Pottery and Porcelain Products," Journal of Information Processing Society of Japan, vol. 55, no. 9, pp. 1992-2007, 2014.

(4) A. Ross and A. K. Jain, "Multimodal Biometrics: An overview," Proc. of 12th European Signal Processing Conference (EUSIPCO), pp. 1221-1224, 2004.

(5) M. Fujikawa, J. Kouki, and S. Fuchi, "A Study on the Multimodal Artifact Metrics and its Application Possibility (Application for Synthetic Resin Products)," The Japanese Journal of the Institute of Industrial Applications Engineers, vol. 5, No. 2, pp. 52-62, 2017.

(6) L. M. Kandpal, S. Lohumi, M. S. Kim, J-S. Kang, and B-K.Cho, "Near-infrared hyperspectral imaging system coupled with multivariate methods to predict viability and vigor in muskmelon seeds," Elsevier Sensors and Actuators B: Chemical, pp. 534-544, 2016.

(7) Y. Suzuki, "Popular science: Kidorui-no-hanashi". (Japanese text). Shokabo publishing Co., Ltd, pp. 144-146. 